\title{
ON THE ZEROS OF SELF-RECIPROCAL POLYNOMIALS SATISFYING CERTAIN COEFFICIENT CONDITIONS
}

\author{
SeOn-Hong Kim* And Jung Hee Lee
}

\begin{abstract}
Kim and Park investigated the distribution of zeros around the unit circle of real self-reciprocal polynomials of even degrees with five terms, where the absolute value of middle coefficient equals the sum of all other coefficients. In this paper, we extend some of their results to the same kinds of polynomials with arbitrary many nonzero terms.
\end{abstract}

\section{Introduction and statement of results}

It what follows, $U$ denotes the unit circle and $n$ is a positive integer. All polynomials in this paper will be assumed to have real coefficients. A polynomial $P(z)$ of degree $n$ is said to be self-inversive if it satisfies $P(z)= \pm z^{n} P(1 / z)$. In particular, if $P(z)=z^{n} P(1 / z), P(z)$ is called self-reciprocal. Thus the zeros of a self-reciprocal polynomial either lie on $U$ or occur in pairs conjugate to $U$. Since the class of self-inversive polynomials of degree $n$ includes polynomials of degree $n$ which have all their zeros on $U$, it is interesting to mention the condition for a self-reciprocal polynomial having all its zeros on $U$. There have been a number of literatures (see [1]-[2] and [4]-[12]) about the distribution of zeros of self-reciprocal polynomials.

The simple self-reciprocal polynomials

$$
A z^{2 m} \pm 2 A z^{m}+A
$$

have all their zeros on $U$. This indicates that zeros of self-reciprocal polynomials (where the absolute value of the middle coefficient equals the sum of all other coefficients) may behave similarly. With this motivation, Kim and Park [5] investigated the distribution of zeros around $U$ of such self-reciprocal polynomials of even degrees with five terms. Our purpose in this paper is to extend some of their results to polynomials with arbitrary many nonzero terms. For

Received April 6, 2009; Revised November 19, 2009.

2000 Mathematics Subject Classification. Primary 30C15; Secondary 26C10.

Key words and phrases. self-reciprocal polynomials, zeros, unit circle.

* This research was supported by the Sookmyung Women's University Research Grants 2009 .

(C)2010 The Korean Mathematical Society 
$m$ a positive integer, we consider self-reciprocal polynomials

$$
P(z)=\sum_{k=0}^{2 m} a_{k} z^{k}, \quad a_{2 m}>0,
$$

where the absolute value of middle coefficient equals the sum of all other coefficients, that is,

$$
\left|a_{m}\right|=\sum_{k=0}^{2 m} a_{k}-a_{m}
$$

In addition, we assume that the coefficients strictly between the leading and the middle term have the same signs. More specifically, we investigate the distribution of zeros of $P(z)$ around $U$ when

(A) $a_{m} \geq 0$, where $a_{k} \geq 0$ for $1 \leq k \leq m-1$,
(B) $a_{m}<0$, where $a_{k} \geq 0$ for $1 \leq k \leq m-1$,
(C) $a_{m} \geq 0$, where $a_{k} \leq 0$ for $1 \leq k \leq m-1$,
(D) $a_{m}<0$, where $a_{k} \leq 0$ for $1 \leq k \leq m-1$.

These four types of polynomials generalize the polynomials studied in [5] in the case when the coefficients strictly between the leading and the middle term have the same signs. Firstly, we may check that all zeros of $P(z)$ in cases $(\mathrm{C})$ and (D) lie on $U$. This is an easy consequence of Theorem 1 of [8] which is the following.

Theorem 1. If $P(z)=\sum_{k=0}^{n} a_{k} z^{k}$ is self-inversive and

$$
\left|a_{n}\right| \geq \frac{1}{2} \sum_{k=0}^{n-1} a_{k}
$$

holds, then all zeros of $P(z)$ are on $U$.

In fact, one can reformulate the coefficient condition (1) as

$$
\frac{\left|a_{m}\right|}{2}=\sum_{k=0}^{m-1} a_{k}=a_{0}+\sum_{k=1}^{m-1} a_{k}
$$

and in cases $(\mathrm{C})$ and $(\mathrm{D})$

$$
\left|a_{0}\right|=a_{0}=-\sum_{k=1}^{m-1} a_{k}+\frac{\left|a_{m}\right|}{2}=\sum_{k=1}^{m-1}\left|a_{k}\right|+\frac{\left|a_{m}\right|}{2}=\frac{1}{2} \sum_{k=1}^{2 m-1}\left|a_{k}\right| .
$$

Thus

$$
\left|a_{0}\right|=\frac{1}{2} \sum_{k=0}^{2 m-1}\left|a_{k}\right|
$$

is exactly the condition (2) with equality. Hence we only need to prove cases (A) and (B). In fact, we will prove Theorem 2 below in Section 2. 
Theorem 2. Let $d$ be the greatest common divisor of positive integers $k$ among

$$
1,2, \ldots, m-1, m+1, \ldots, 2 m
$$

where $a_{k} \neq 0$. Then we have the following.

(a) If $d \mid m$, then $P(z)$ in case (A) has no zeros on $U$.

(b) If $d \mid m$, then, in case $(\mathrm{B}), P(z)$ has exactly $d$ zeros on $U$ without counting multiplicities. Such zeros are the d-th roots of 1.

(c) If $d \nmid m$, then, in cases $(\mathrm{A})$ and $(\mathrm{B}), d$ is even and $P(z)$ has exactly $d / 2$ zeros on $U$ without counting multiplicities. Such zeros are the $d / 2$-th roots of -1 for $(\mathrm{A})$ and the $d / 2$-th roots of 1 for $(\mathrm{B})$, respectively.

\section{Proof}

Proof of Theorem 2. Firstly, we prove (a) and the case (A) of (c). We may assume that $a_{m}>0$ because $P(z)$ is the zero polynomial when $a_{m}=0$. For $\epsilon>0$, we define the polynomial

$$
P_{\epsilon}(z)=\sum_{k=0}^{m-1} a_{k} z^{k}+\left(a_{m}+\epsilon\right) z^{m}+\sum_{k=m+1}^{2 m} a_{k} z^{k} .
$$

It follows from the triangle inequality and from the positivity of $a_{m}$ that, for $|z|=1$

$$
\left|P_{\epsilon}(z)\right| \geq\left(a_{m}+\epsilon\right)-2 \sum_{k=0}^{m-1} a_{k}=\epsilon>0 .
$$

Thus $P_{\epsilon}(z)$ does not have a zero on $U$, and so $P_{\epsilon}(z)$ has exactly $m$ zeros strictly inside $U$, say $\alpha_{1}, \ldots, \alpha_{m}$. Assume on the contrary to (a) that $P$ has at least one zero on $U$. Then for at least one $j$ we have

$$
\alpha_{j} \rightarrow z_{j}:=e^{i \theta_{j}} \in U\left(\theta_{j} \in \mathbb{R}\right) \text { as } \epsilon \rightarrow 0
$$

and $P\left(z_{j}\right)=0$. Since $P\left(z_{j}\right)=0$, we have

$$
\sum_{k=0}^{2 m} a_{k} e^{i k \theta_{j}}=0
$$

and thus

$$
\begin{aligned}
0=P\left(z_{j}\right) & =e^{i m \theta_{j}}\left(\sum_{k=0}^{m-1} a_{k}\left(e^{i(m-k) \theta_{j}}+e^{-i(m-k) \theta_{j}}\right)+a_{m}\right) \\
& =e^{i m \theta_{j}}\left(\sum_{k=0}^{m-1} 2 a_{k} \cos (m-k) \theta_{j}+\sum_{k=0}^{m-1} 2 a_{k}\right) .
\end{aligned}
$$

Therefore

$$
\sum_{k=0}^{m-1} a_{k}\left(\cos (m-k) \theta_{j}+1\right)=0 .
$$


Since all terms of the above sum are nonnegative, they must be zero. Hence $a_{k} \neq 0(k=0,1, \ldots, m-1)$ implies $\cos (m-k) \theta_{j}+1=0$. Using this for $k=0$ and another $k=1,2, \ldots, m-1$, one can conclude that

$$
k \theta_{j} \equiv 0(\bmod 2 \pi)
$$

for all $k, 0 \leq k \leq 2 m, k \neq m$ with $a_{k} \neq 0$. Let $d$ be the greatest common divisor of those positive integers $k$ among

$$
1,2, \ldots, m-1, m+1, \ldots, 2 m
$$

for which $a_{k} \neq 0$. Then $z_{j}=e^{i \theta_{j}}$ is a $d$-th root of unity. In case of $d \mid m$,

$$
P\left(z_{j}\right)=2 \sum_{k=0}^{m-1} a_{k}+a_{m} z_{j}^{m}=a_{m}\left(1+z_{j}^{m}\right)=2 a_{m} \neq 0
$$

which is a contradiction, proving (a). For the proof of the case (A) of (c), we suppose that $d \nmid m$ and $m=d k+r$ for some integers $k, r$ with $1 \leq r \leq d-1$. Then $d$ must be even since, for $d$ odd, $d \mid 2 m$ and so $d \mid m$. Also $d \mid 2 m$ implies that $d \mid 2 r$. Put $2 r=d u$ for some positive integer $u$. Then $d u / 2<d$ and so $u=1$, that is, $d=2 r$. So

$$
P\left(z_{j}\right)=2 \sum_{k=0}^{m-1} a_{k}+a_{m} z_{j}^{m}=a_{m}\left(1+z_{j}^{m}\right)=a_{m}\left(1+z_{j}^{r}\right)=a_{m}\left(1+z_{j}^{d / 2}\right) .
$$

Since the $d / 2$-th roots of -1 are contained in the $d$-th roots of unity, $P(z)$ has zeros on $U$, and it has exactly $d / 2$ zeros on $U$ without counting multiplicities. Such zeros are the $d / 2$-th roots of -1 . Next we prove (b) and the case (B) of (c) even though the proofs for these are very similar to the above. For $\epsilon>0$, we define the polynomial

$$
P_{\epsilon, 1}(z)=\sum_{k=0}^{m-1} a_{k} z^{k}+\left(a_{m}-\epsilon\right) z^{m}+\sum_{k=m+1}^{2 m} a_{k} z^{k}
$$

It follows from the triangle inequality and from the negativeness of $a_{m}$ that, for $|z|=1$,

$$
\left|P_{\epsilon, 1}(z)\right| \geq\left(-a_{m}+\epsilon\right)-2 \sum_{k=0}^{m-1} a_{k}=\epsilon>0 .
$$

Thus $P_{\epsilon, 1}(z)$ does not have a zero on $U$, and so $P_{\epsilon, 1}(z)$ has exactly $m$ zeros strictly inside $U$, say $\beta_{1}, \ldots, \beta_{m}$. Suppose that $P$ has at least one zero on $U$. Then for at least one $j$ we have

$$
\beta_{j} \rightarrow w_{j}:=e^{i \rho_{j}} \in U\left(\rho_{j} \in \mathbb{R}\right) \text { as } \epsilon \rightarrow 0
$$

and $P\left(w_{j}\right)=0$. Since $P\left(w_{j}\right)=0$, we have

$$
\sum_{k=0}^{2 m} a_{k} e^{i k \rho_{j}}=0
$$


We follow the process to get (3) in the proof of (a) so that we obtain

$$
k \rho_{j} \equiv 0(\bmod 2 \pi)
$$

for all $k, 0 \leq k \leq 2 m, k \neq m$ with $a_{k} \neq 0$. So $w_{j}=e^{i \rho_{j}}$ is a $d$-th root of unity, where $d$ was the greatest common divisor of positive integers among all $k$ 's, where $0 \leq k \leq 2 m, k \neq m$ with $a_{k} \neq 0$. In case of $d \mid m$,

$$
P\left(w_{j}\right)=2 \sum_{k=0}^{m-1} a_{k}+a_{m} w_{j}^{m}=a_{m}\left(w_{j}^{m}-1\right) .
$$

Thus $P(z)$ has zeros on $U$, and it has exactly $d$ zeros on $U$ that are the $d$-th roots of unity. We now suppose that $d \nmid m$ and $m=d k+r$ for some integers $k, r$ with $1 \leq r \leq d-1$. Then we note that $d=2 r$ as before. So

$$
P(w)=2 \sum_{k=0}^{m-1} a_{k}+a_{m} w^{m}=a_{m}\left(w^{m}-1\right)=a_{m}\left(w^{r}-1\right) .
$$

Since the $d / 2$-th roots of unity are contained in the $d$-th roots of unity, $P(z)$ has exactly $d / 2$ zeros on $U$ without counting multiplicities. Such zeros are the $d / 2$-th roots of unity. This completes the proof of Theorem 2 .

Acknowledgment. The authors wish to thank the referee for many useful suggestions on a earlier version of this paper.

\section{References}

[1] G. Ancochea, Zeros of self-inversive polynomials, Proc. Amer. Math. Soc. 4 (1953), 900-902.

[2] F. F. Bonsall and M. Marden, Zeros of self-inversive polynomials, Proc. Amer. Math. Soc. 3 (1952), 471-475.

[3] A. Cohn, Über die Anzahl der Wurzeln einer algebraischen Gleichung in einem Kreise, Math. Z. 14 (1922), no. 1, 110-148.

[4] S.-H. Kim, The zeros of certain family of self-reciprocal polynomials, Bull. Korean Math. Soc. 44 (2007), no. 3, 461-473.

[5] S.-H. Kim and C. W. Park, On the zeros of certain self-reciprocal polynomials, J. Math. Anal. Appl. 339 (2008), no. 1, 240-247.

[6] P. Lakatos, On zeros of reciprocal polynomials, Publ. Math. Debrecen 61 (2002), no. 3-4, 645-661.

[7] P. Lakatos and L. Losonczi, Circular interlacing with reciprocal polynomials, Math. Inequal. Appl. 10 (2007), no. 4, 761-769.

[8] _ Self-inversive polynomials whose zeros are on the unit circle, Publ. Math. Debrecen 65 (2004), no. 3-4, 409-420.

[9] , On zeros of reciprocal polynomials of odd degree, J. Inequal. Pure Appl. Math. 4 (2003), no. 3, Article 60, 8 pp.

[10] L. Losonczi, On reciprocal polynomials with zeros of modulus one, Math. Inequal. Appl. 9 (2006), no. 2, 289-298.

[11] L. Losonczi and A. Schinzel, Self-inversive polynomials of odd degree, Ramanujan J. 14 (2007), no. 2, 305-320.

[12] A. Schinzel, Self-inversive polynomials with all zeros on the unit circle, Ramanujan J. 9 (2005), no. 1-2, 19-23. 
SEON-HONG KIM

Department of Mathematics

SOOKMYUng Women's UNIVERSITY

SEOUl 140-742, KoreA

E-mail address: shkim17@sookmyung.ac.kr

Jung HeE LeE

Department of Mathematics

SOOKMYUNG WOMEN'S UNIVERSITY

SEOUl 140-742, KoreA

E-mail address: dosk12@hanmail.net 